\title{
Teleportation of continuous quantum variables using squeezed-state entanglement
}

Kok Win Goh, Chin-Wen Chou, Tiancai Zhang, Peter Lodahl, H. Jeff Kimble

Kok Win Goh, Chin-Wen Chou, Tiancai Zhang, Peter Lodahl, H. Jeff Kimble, "Teleportation of continuous quantum variables using squeezed-state entanglement," Proc. SPIE 5161, Quantum Communications and Quantum Imaging, (3 February 2004); doi: 10.1117/12.508566

SPIE Event: Optical Science and Technology, SPIE's 48th Annual Meeting, 2003, San Diego, California, United States 


\title{
Teleportation of continuous quantum variables using squeezed-state entanglement
}

\author{
K. W. Goh ${ }^{a}$, C. W. Chou ${ }^{a}$, T. C. Zhang ${ }^{b}$, P. Lodahl ${ }^{c}$, and H. J. Kimble ${ }^{a}$ \\ ${ }^{a}$ California Institute of Technology 12-33, Pasadena, California 91125, USA \\ ${ }^{b}$ Shanxi University, Taiyuan 030006, China \\ ${ }^{c}$ University of Twente, P. O. Box 217, 7500 AE Enschede, The Netherlands
}

\section{PRESENTATION SUMMARY}

Quantum teleportation, the "disembodied transport" of quantum states as described in the work of Bennett $e t$ al. ${ }^{1}$ is made possible by using both classical and quantum shared resources. In our research lab, we create Einstein-Podolsky-Rosen (EPR) entanglement ${ }^{2}$ by combining squeezed-states of light at a beamsplitter, thus providing the necessary quantum resource. Figure 1 shows the main components required for teleportation as relevant to our particular laboratory, showing both the classical and quantum communication channels shared by Alice (the sender) and Bob (the receiver), the input state $\left|\psi_{i n}\right\rangle$ to be teleported, and the teleported output state $\rho_{\text {out }}$. The figure also shows the orthogonal $x$ and $p$ quadratures that are measured by Alice using standard homodyne techniques, and that constitute the set of continuous variables involved in the teleportation procedure. The theoretical basis for this protocol is described in Ref. 3 with experimental results given in Ref. 4. Ref. 5 provides a detailed description of the entire experimental procedure as well as some recent results. For an overview of recent research developments in quantum continuous variables, see Ref. 6 .

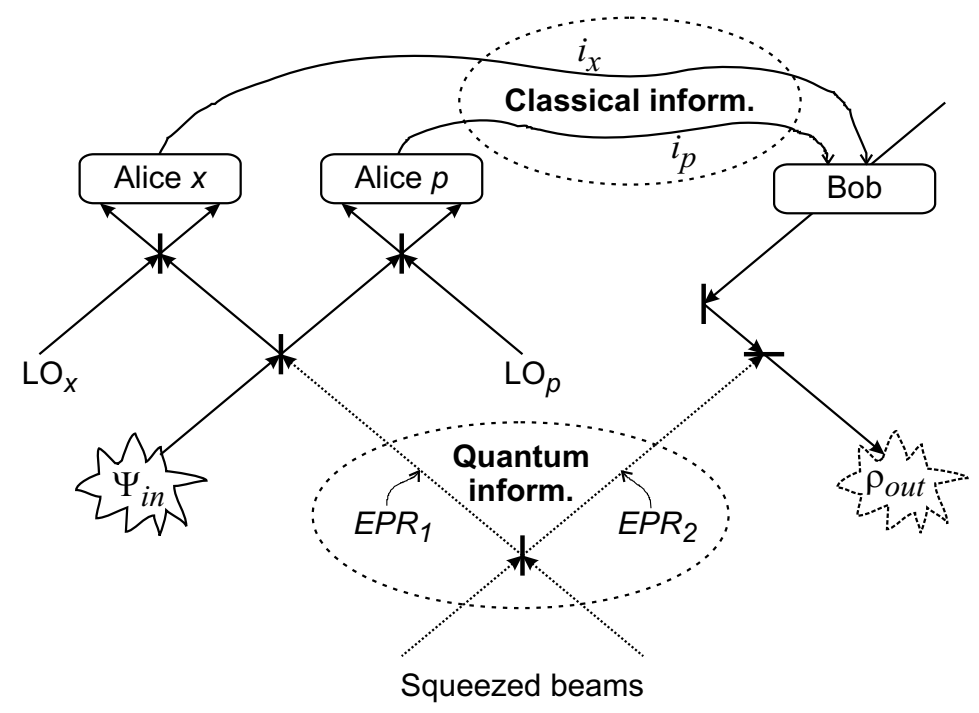

Figure 1. Main parts in the teleportation procedure, showing Alice and Bob's classical and quantum shared resources. Classical information is transfered in the form of photocurrents $\left(i_{x}, i_{p}\right)$, while quantum information is shared via the entangled beams $E P R_{1}$ and $E P R_{2}$. LO: Local Oscillator; Inform: information.

The performance of the teleportation protocol is quantified by the fidelity $F$, which is given by the overlap between $\left|\psi_{\text {in }}\right\rangle$ and $\rho_{\text {out }}$, as discussed in detail in Refs. 7 and 8. Formally, $F=\left\langle\psi_{\text {in }}\left|\rho_{\text {out }}\right| \psi_{\text {in }}\right\rangle$. In order to better understand what limits $F$, we investigated various loss factors under realistic laboratory conditions by using a detailed model of the experiment that includes essentially all dominant loss mechanisms. These losses can

Send correspondence to K. W. Goh (email: kgoh@its.caltech.edu) 
be loosely grouped into three areas, namely limited degrees of squeezing, inefficient detection of the input and entangled states including imperfect spatial mode-matching of the light beams, and imperfect determination of the $x$ and $p$ quadratures at Alice's and Bob's stations (mainly due to servo-lock phase fluctuations and innaccurate data transfer in the classical communication channel.)

The squeezed light beams are generated via parametric down-conversion in a subthreshold Optical Parametric Oscillator (OPO). ${ }^{9}$ The degree of squeezing generated in this way is limited by losses in the OPO cavity, which in our case comes mainly from absorption in our nonlinear potassium niobate $\left(\mathrm{KNbO}_{3}\right)$ crystal. In addition to passive losses $(\sim 0.3-0.5 \%), \mathrm{KNbO}_{3}$ also suffers from a process termed "blue-light-induced infrared absorption" (BLIIRA). ${ }^{10}$ This nonlinear loss factor is often much higher $(\gtrsim 2 \%)$ than the passive losses at normal pump powers for the OPO. We observe $\sim 3.7 \mathrm{~dB}$ of squeezing below the vacuum noise level available for teleportation (measured degree of squeezing at the sending and receiving stations.) This limited degree of squeezing proves to be the main limitation to achieving higher fidelity. It must also be noted that the measured degree of squeezing is lower than predicted values based on independent measurements of the parameters of our OPO, including BLIIRA. Indeed, we expect almost $1 \mathrm{~dB}$ more squeezing ( $\gtrsim 4.5 \mathrm{~dB}$ below the vacuum noise level) with the experimental parameters relevant to these data.

To date, our best data set gives a raw (inferred directly from measurements of the noise properties of $\rho_{\text {out }}$ with no corrections) fidelity for the teleportation of coherent states of $F_{M}=0.61 \pm 0.02$, which is significant as it is above $F_{C}=0.50$, the maximum achievable fidelity with no entanglement. ${ }^{7,8}$ By contrast, based on independently measured degrees of squeezing and detection efficiencies, our model predicts that we should be able to reach $F_{P}=0.69$. This discrepancy between the measured fidelities $\left\{F_{m}\right\}$, and predicted fidelities $\left\{F_{p}\right\}$, is demonstrated in Fig. 2 where we plot $F$ versus the OPO pump power for a particular data run. The dots with error-bars represent $\left\{F_{m}\right\}$ and the triangles are $\left\{F_{p}\right\}$ based on independent squeezing and detection loss measurements.

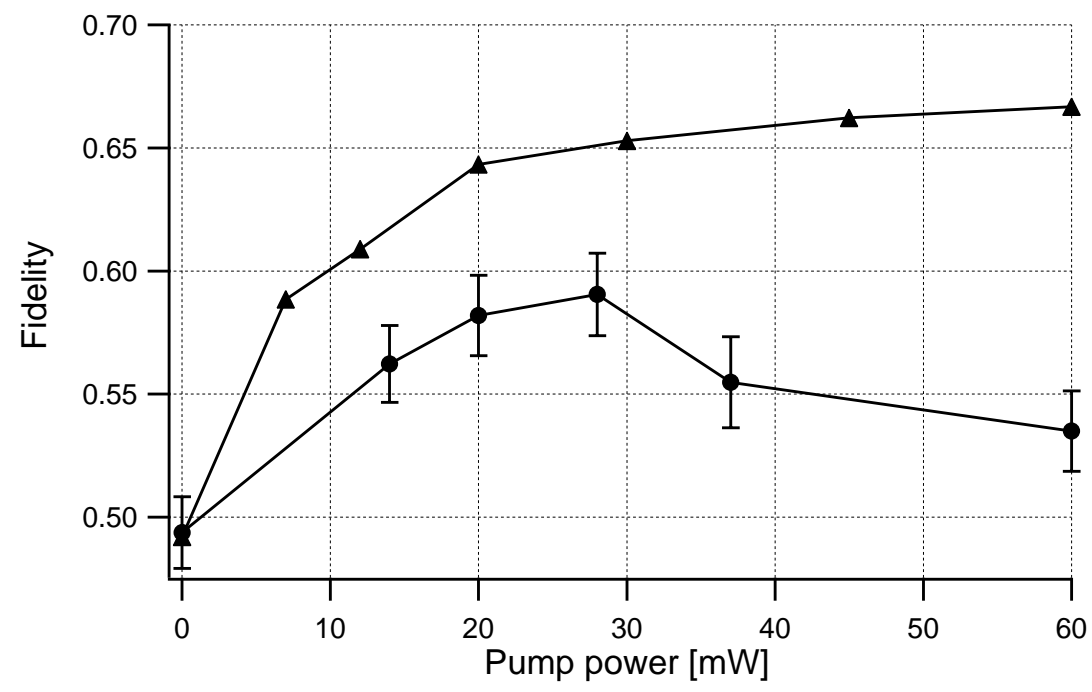

Figure 2. Fidelity as a function of the OPO pump power. Dots are the experimentally measured fidelities $\left\{F_{m}\right\}$, and triangles are the expected fidelities given the measured degrees of squeezing $\left\{F_{p}\right\}$ for this data set. The data points have been connected to ease viewing.

We at once notice that the $\left\{F_{m}\right\}$ are always lower than $\left\{F_{p}\right\}$ regardless of OPO pump power. However, the measured and predicted fidelities exhibit diferrent trends. $\left\{F_{p}\right\}$ increases monotonically with pump power as may be expected although it does level-off at pump powers above $60 \mathrm{~mW}$, while $\left\{F_{m}\right\}$ reaches a maximum (for this data set) at the rather low pump level of $\sim 30 \mathrm{~mW}$ and degrades at higher pump levels. It must be noted that the $\left\{F_{p}\right\}$ are calculated by assuming the $(x, p)$ phases are correct. Our model does allow us to estimate 
the deterioration in fidelity with imprecise determination of $(x, p)$. Although the losses that arise from $(x, p)$ phase fluctuations can be significant, our best estimates imply that they do not account for the discrepancy in our predicted and measured fidelities.

Current efforts as well as speculations centered on the ongoing attempt to understand the discrepancy shown in Fig. 2 will be presented.

Keywords: Quantum teleportation, entanglement, squeezing, nonlinear crystals

\section{ACKNOWLEDGMENTS}

This work was supported by the National Science Foundation, by the Office of Naval Research, and by the Caltech MURI Center for Quantum Networks.

\section{REFERENCES}

1. C. H. Bennett, G. Brassard, C. Crepeau, R. Jozsa, A. Peres, and W. K. Wootters, "Teleporting an unknown quantum state via dual classical and Einstein-Podolsky-Rosen channels," Phys. Rev. Lett. 70, pp. 1895-1899, 1993.

2. A. Einstein, B. Podolsky, and N. Rosen, "Can quantum-mechanical description of physical reality be considered complete?" Phys. Rev. 47, pp. 777-780, 1935.

3. S. L. Braunstein and H. J. Kimble, "Teleportation of continuous quantum variables," Phys. Rev. Lett. 80, pp. 869-872, 1998.

4. A. Furusawa, J. L. Sørensen, S. L. Braunstein, C. A. Fuchs, H. J. Kimble, and E. S. Polzik, "Unconditional quantum teleportation," Science 282, pp. 706-709, 1998.

5. T. C. Zhang, K. W. Goh, C. W. Chou, P. Lodahl, and H. J. Kimble, "Quantum teleportation of light beams," Phys. Rev. A 67, art. 033802, 2003.

6. S. L. Braunstein and A. K. Pati, eds., Quantum Information Theory with Continuous Variables, Kluwer Academic Publishers, Dodrecht, 2003.

7. S. L. Braunstein, C. A. Fuchs, and H. J. Kimble, "Criteria for continuous-variable quantum teleportation," J. Mod. Opt. 47, pp. 267-278, 2000.

8. S. L. Braunstein, C. A. Fuchs, H. J. Kimble, and P. van Loock, "Quantum versus classical domains for teleportation with continuous variables," Phys. Rev. A 64, art. 022321, 2001.

9. E. S. Polzik, J. Carri, and H. J. Kimble, "Spectroscopy with squeezed light," Phys. Rev. Lett. 68, pp. 30203023, 1992; "Atomic Spectroscopy with squeezed light for sensitivity beyond the vacuum-state limit," Appl. Phys. B 55, pp. 279-290, 1992.

10. H. Mabuchi, E. S. Polzik, and H. J. Kimble, "Blue-light-induced infrared absorption in $\mathrm{KNbO}_{3}$," J. Opt. Soc. Am. B 11, pp. 2023-2029, 1994. 